\title{
Practical Beam-Beam Tune Shift Formulae for Simulation Cross-Check*
}

\author{
A. Valishev, FNAL, Batavia IL, 60510 USA
}

December 9, 2013

\begin{abstract}
In this note, practical analytical formulae for head-on beam-beam tune shift are derived for the case of arbitrary crossing angle and non-negligible hourglass effect. The formulae are evaluated using numerical integration, and the results used to cross-check with the particle tracking code Lifetrac.
\end{abstract}

\section{Introduction}

The general approach to derive the betatron tune shift for a particle with small amplitude passing through the electromagnetic field of a counter-propagating beam bunch follows that of [1] and [2]. The former case treats the hourglass effect, and the latter provides formulae for bunches colliding at an angle. Our derivation combines the two approaches.

\section{Tune Shift Formulae}

The expressions for electromagnetic field of a 3D Gaussian ellipsoid are [3]:

$$
\begin{aligned}
& E_{x}=\frac{e N \gamma}{2 \varepsilon_{0} \sqrt{\pi}^{3}} x \int_{0}^{\infty} d w \frac{\exp \left(-\frac{x^{2}}{2 \sigma_{x}^{2}+w}-\frac{y^{2}}{2 \sigma_{y}^{2}+w}-\frac{\gamma^{2}(z-c t)^{2}}{2 \gamma^{2} \sigma_{z}^{2}+w}\right)}{\left(2 \sigma_{x}^{2}+w\right)^{3 / 2}\left(2 \sigma_{y}^{2}+w\right)^{1 / 2}\left(2 \gamma^{2} \sigma_{z}^{2}+w\right)^{1 / 2}} \\
& E_{y}=\frac{e N \gamma}{2 \varepsilon_{0} \sqrt{\pi}^{3}} y \int_{0}^{\infty} d w \frac{\exp \left(-\frac{x^{2}}{2 \sigma_{x}^{2}+w}-\frac{y^{2}}{2 \sigma_{y}^{2}+w}-\frac{\gamma^{2}(z-c t)^{2}}{2 \gamma^{2} \sigma_{z}^{2}+w}\right)}{\left(2 \sigma_{x}^{2}+w\right)^{1 / 2}\left(2 \sigma_{y}^{2}+w\right)^{3 / 2}\left(2 \gamma^{2} \sigma_{z}^{2}+w\right)^{1 / 2}} \\
& B_{x}=-\frac{E_{y}}{c}, B_{y}=\frac{E_{x}}{c}
\end{aligned}
$$

Here $N$ is the number of particles in the bunch, $x, y, z$ are the Cartesian horizontal, vertical and longitudinal coordinates in the system of strong bunch, $\sigma_{x}, \sigma_{y}, \sigma_{z}$ are the horizontal, vertical and longitudinal beam sizes. We denote the coordinates and fields for a particle in the weak bunch in the system of that bunch using the ' sign. If the weak bunch is moving at a horizontal angle $\theta$ with respect to the strong bunch axis, the coordinate transformations are:

$$
\left\{\begin{array}{l}
x=x^{\prime} \cos (\theta)+z^{\prime} \sin (\theta) \\
y=y^{\prime} \\
z=z^{\prime} \cos (\theta)-x^{\prime} \sin (\theta)
\end{array}\right.
$$

The equations of motion for a particle in the weak beam are:

$$
\left\{\begin{array}{l}
x(t)=-c t \sin (\theta)+x_{0} \\
y(t)=y_{0} \\
z(t)=-c t \cos (\theta)+z_{0}
\end{array}\right.
$$

\footnotetext{
* *Fermi Research Alliance, LLC operates Fermilab under contract No. DE-AC02-07CH11359 with the U.S. Department of Energy. This work was partially supported by the US LHC Accelerator Research Program (LARP).
} 
The horizontal and vertical Lorentz force for a particle in the weak beam is:

$$
\left\{\begin{array}{l}
F_{x}^{\prime}=F_{x}=e\left(E_{x}-v_{z} B_{y}\right)=e E_{x}(1+\cos (\theta)) \\
F_{y}^{\prime}=F_{y}=e\left(E_{y}+v_{z} B_{x}\right)=e E_{y}(1+\cos (\theta))
\end{array}\right.
$$

Tune shift for a particle in the weak beam due to a thin transverse focusing element is:

$$
\left\{\begin{array}{l}
\Delta Q_{x}=\frac{\beta_{x}}{4 \pi} G_{x}=\frac{\beta_{x}}{4 \pi} \frac{1}{p_{0}} \frac{\partial \Delta p_{x}^{\prime}}{\partial x^{\prime}}=\frac{\beta_{x}}{4 \pi} \frac{d t}{p_{0}} \frac{\partial \Delta F_{x}^{\prime}}{\partial x^{\prime}} \\
\Delta Q_{y}=\frac{\beta_{y}}{4 \pi} G_{y}=\frac{\beta_{y}}{4 \pi} \frac{1}{p_{0}} \frac{\partial \Delta p_{y}^{\prime}}{\partial x^{\prime}}=\frac{\beta_{y}}{4 \pi} \frac{d t}{p_{0}} \frac{\partial \Delta F_{y}^{\prime}}{\partial y^{\prime}}
\end{array}\right.
$$

Assuming no external fields in the interaction region, and the minimum beta-function coinciding with the $z=0$ position, one can express the horizontal and vertical beam sizes as functions of $z$ :

$$
\left\{\begin{array}{l}
\sigma_{x}(z)=\sqrt{\varepsilon_{x} \beta_{x}^{*}\left(1+z^{2} / \beta_{x}^{*}\right)} \\
\sigma_{y}(z)=\sqrt{\varepsilon_{y} \beta_{y}^{*}\left(1+z^{2} / \beta_{y}^{*}\right)}
\end{array}\right.
$$

Here $\varepsilon_{x}, \varepsilon_{y}$ are the horizontal and vertical emittance of the strong beam, $\beta_{x}^{*}$ and $\beta_{y}^{*}$ are the horizontal and vertical beta-functions at the IP.

Substituting (2), (3) into (1) and combining (4) and (5), and integrating (5) over $t$, we obtain:

$$
\begin{aligned}
& d z \beta_{x}(z)\left(\cos (\theta)+2 z^{2} \sin (\theta)^{2}\left[\frac{\gamma^{2} z^{2}(\cos (\theta)-1)}{2 \gamma^{2} \sigma_{z}^{2}+w}-\frac{1}{2 \sigma_{x}(z)^{2}+w}\right]\right) \times \\
& \Delta Q_{x}=\frac{r_{0} N}{2 \pi^{3 / 2}}(1+\cos (\theta)) \int_{0}^{\infty} d w \int_{-\infty}^{\infty} \quad \times \frac{\exp \left(-\frac{z^{2} \sin (\theta)^{2}}{2 \sigma_{x}(z)^{2}+w}-\frac{\gamma^{2} z^{2}(1+\cos (\theta))^{2}}{2 \gamma^{2} \sigma_{z}^{2}+w}\right)}{\left(2 \sigma_{x}(z)^{2}+w\right)^{3 / 2}\left(2 \sigma_{y}(z)^{2}+w\right)^{1 / 2}\left(2 \gamma^{2} \sigma_{z}^{2}+w\right)^{1 / 2}} \\
& \Delta Q_{y}=\frac{r_{0} N}{2 \pi^{3 / 2}}(1+\cos (\theta)) \int_{0}^{\infty} d w \int_{-\infty}^{\infty} d z \beta_{y}(z) \frac{\exp \left(-\frac{z^{2} \sin (\theta)^{2}}{2 \sigma_{x}(z)^{2}+w}-\frac{\gamma^{2} z^{2}(1+\cos (\theta))^{2}}{2 \gamma^{2} \sigma_{z}^{2}+w}\right)}{\left(2 \sigma_{x}(z)^{2}+w\right)^{1 / 2}\left(2 \sigma_{y}(z)^{2}+w\right)^{3 / 2}\left(2 \gamma^{2} \sigma_{z}^{2}+w\right)^{1 / 2}}
\end{aligned}
$$

For negligible hourglass effect $\left(\sigma_{z} / \beta_{x, y} \ll 1\right)$ one can neglect the $\sigma_{x}(z)$ and $\sigma_{y}(z)$ substituting them with the constant values $\sigma_{x, y}^{*}=\sqrt{\varepsilon_{x, y} \beta_{x, y}^{*}}$. Then, the integrals can be taken for $\theta=0$, arriving at the well-known expressions:

$$
\begin{aligned}
& \Delta Q_{x}=\frac{r_{0} N \beta_{x}^{*}}{2 \pi \gamma \sigma_{x}^{*}\left(\sigma_{x}^{*}+\sigma_{y}^{*}\right)}=\xi_{x 0} \\
& \Delta Q_{y}=\frac{r_{0} N \beta_{y}^{*}}{2 \pi \gamma \sigma_{y}^{*}\left(\sigma_{x}^{*}+\sigma_{y}^{*}\right)}=\xi_{y 0}
\end{aligned}
$$

For simplicity of the following analysis we introduce the normalized tune shift factors:

$$
R_{x}=\frac{\Delta Q_{x}}{\xi_{x 0}}, R_{y}=\frac{\Delta Q_{y}}{\xi_{y 0}}
$$




\section{Head-on Tune Shift for Round Beams}

Tune shift for round beams in the case of full head-on collision $(\theta=0)$ can be obtained analytically with a much simpler derivation. Noting that for the ultra-relativistic case the longitudinal field component can be neglected, and using the axial symmetry of the system, the expression for the electrical field of a round beam is derived from the Gauss' law:

$$
2 \pi r \cdot E_{r}=\frac{1}{\varepsilon_{0}} \rho(z) d z \int_{0}^{r} \rho_{r}\left(r^{\prime}\right) 2 \pi r^{\prime} d r^{\prime}
$$

Substituting Gaussian longitudinal and transverse densities, we obtain:

$$
E_{r}=\frac{e N}{2 \pi \varepsilon_{0}} \frac{e^{-z^{2} / 2 \sigma_{z}^{2}}}{\sqrt{2 \pi} \sigma_{z}} d z \frac{1}{r}\left[1-e^{-r^{2} / 2 \sigma^{2}}\right]
$$

Then, using $F_{r}=2 e E_{r}$ and (5), the tune shift is:

$$
\begin{gathered}
\Delta Q_{x}=\Delta Q_{y}=\left.\frac{r_{0} N}{2 \pi \gamma} \int_{-\infty}^{\infty} \frac{d z}{\sqrt{2 \pi} \sigma_{z}} \beta(z) e^{-z^{2} / 2 \sigma_{z}^{2}} \frac{\partial}{\partial x}\left[\frac{1-e^{-r^{2} / 2 \sigma(z)^{2}}}{r}\right]\right|_{r=0}= \\
\frac{r_{0} N}{4 \pi \gamma} \int_{-\infty}^{\infty} \frac{d z}{\sqrt{2 \pi} \sigma_{z}} \frac{\beta(z)}{\sigma(z)^{2}} e^{-z^{2} / 2 \sigma_{z}^{2}}=\frac{r_{0} N}{4 \pi \gamma} \int_{-\infty}^{\infty} \frac{d z}{\sqrt{2 \pi} \sigma_{z}} \frac{\beta(z)}{\varepsilon \beta(z)} e^{-z^{2} / 2 \sigma_{z}^{2}}=\frac{r_{0} N}{4 \pi \gamma \varepsilon}
\end{gathered}
$$

Hence, the head-on tune shift is not affected by hourglass effect.

\section{Cross-Check with Lifetrac}

The formulae (7) were integrated numerically for a number of beam parameters. Below the results of these calculations are compared with the tune shifts calculated with the particle tracking code Lifetrac. The tune shift calculation in Lifetrac was performed via the normal mode analysis, in which a one turn linear map of the machine is calculated by tracking a sample particle with varying initial conditions and the subsequent derivation of a linearized map. This procedure has limited precision, which explains the observed minor difference with the analytical calculation. Otherwise, the agreement is very good.

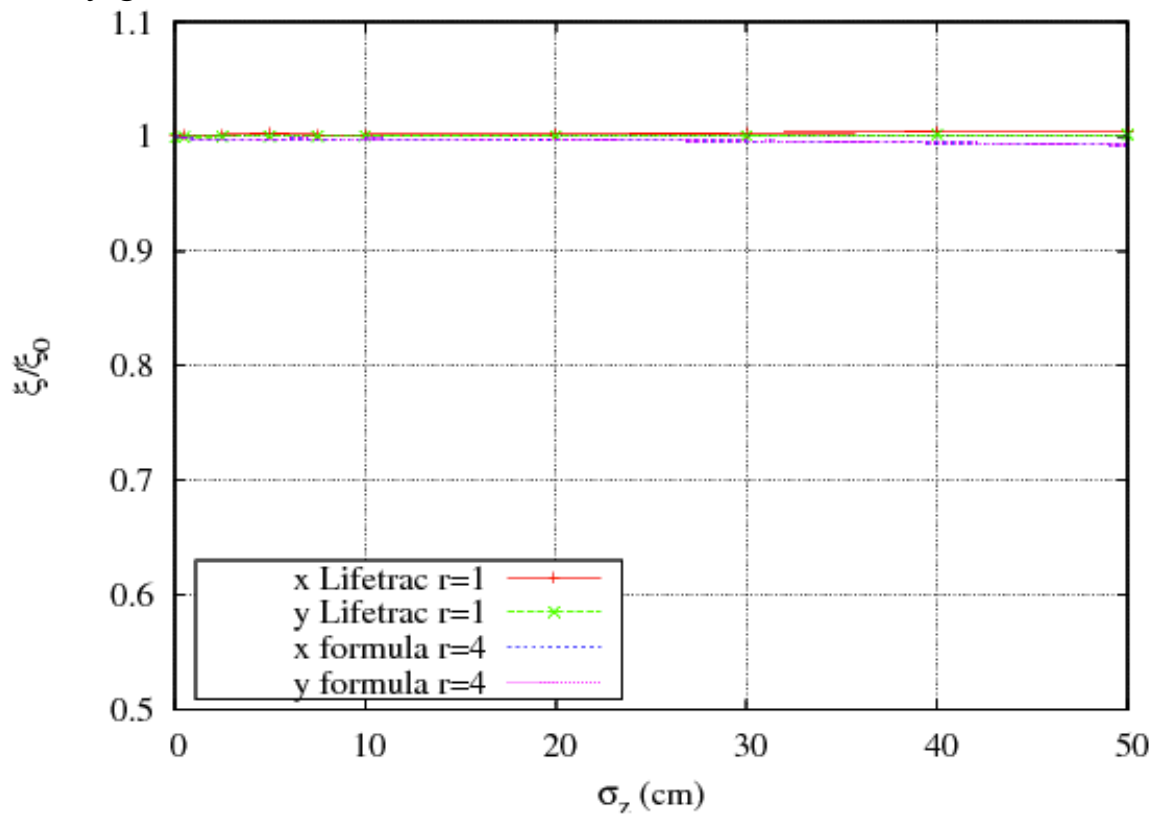

Figure 1: Tune shift factor vs. bunch length for $\theta=0$. Round beam $\beta_{\mathrm{x}}=15 \mathrm{~cm}, \beta_{\mathrm{y}}=15 \mathrm{~cm}, \varepsilon_{\mathrm{x}}=\varepsilon_{\mathrm{y}}$. 
FERMILAB-TM-2573-APC

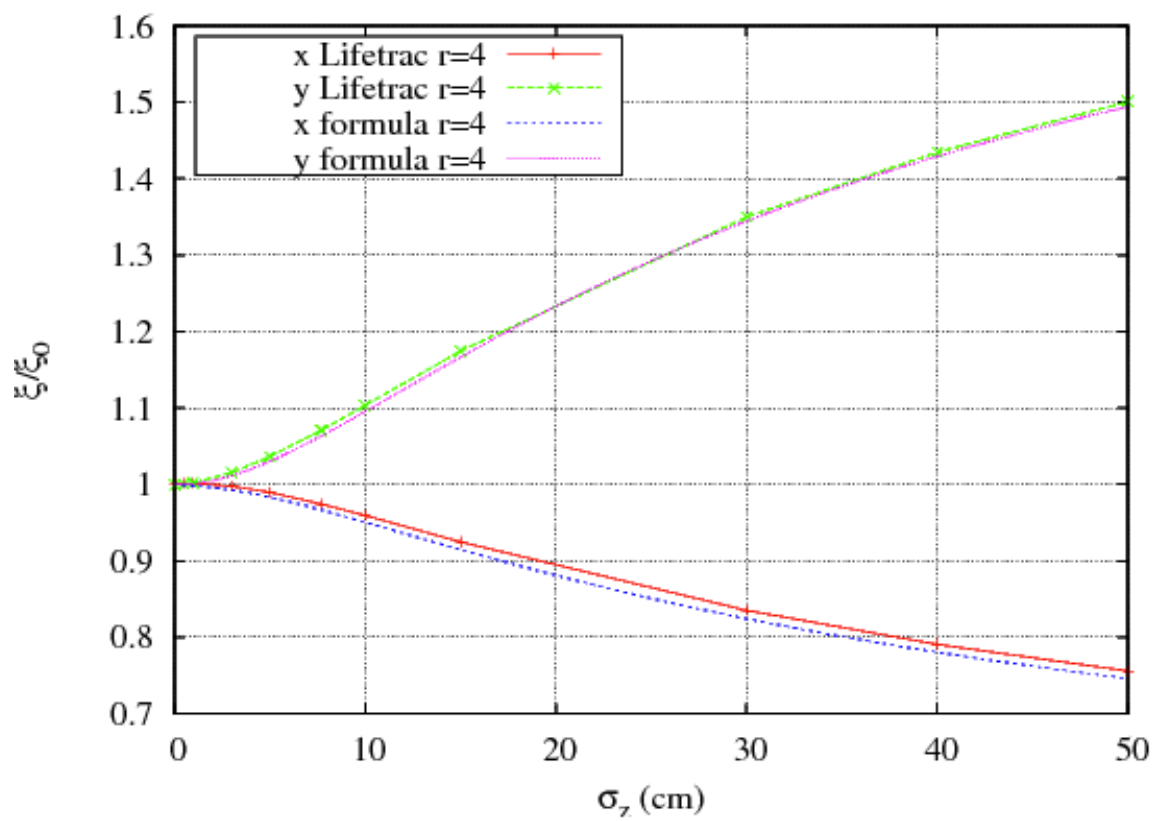

Figure 2: Tune shift factor vs. bunch length for $\theta=0$. Flat beam $\beta_{\mathrm{x}}=30 \mathrm{~cm}, \beta_{\mathrm{y}}=7.5 \mathrm{~cm}, \varepsilon_{\mathrm{x}}=\varepsilon_{\mathrm{y}}$.

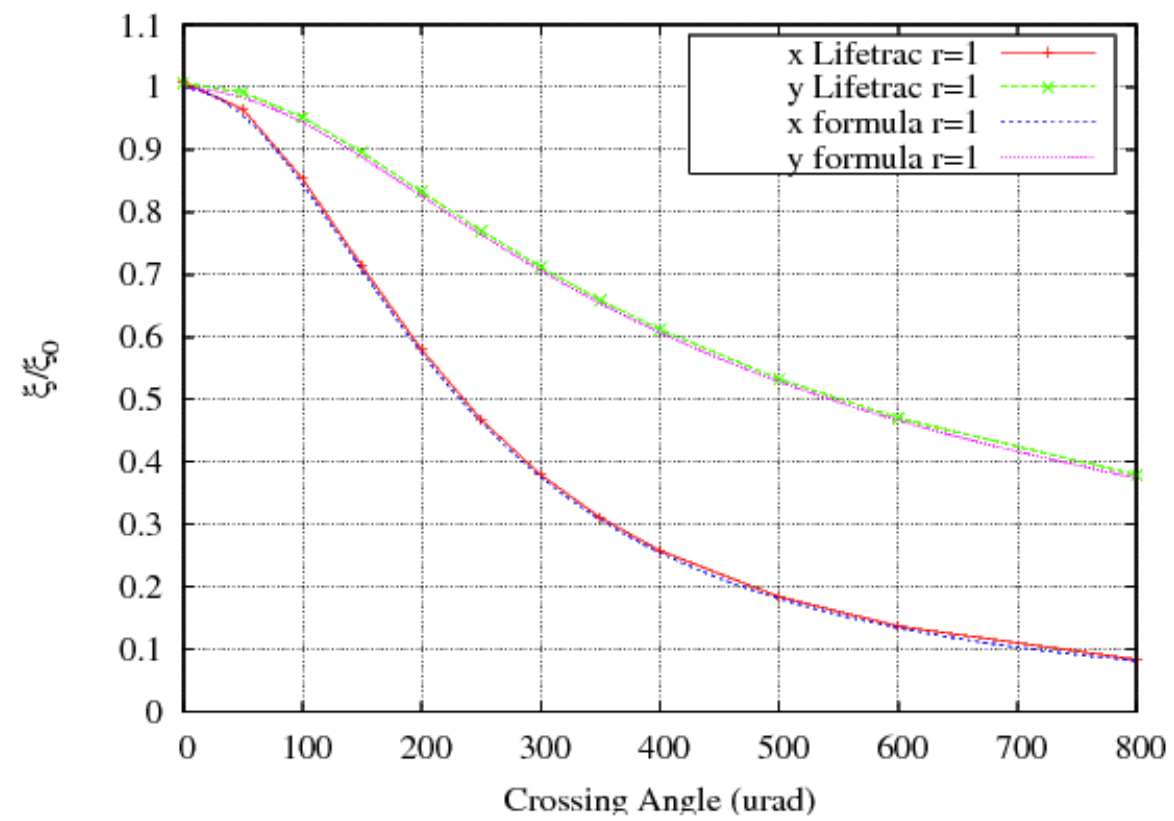

Figure 3: Tune shift factor vs. crossing angle for $\sigma_{\mathrm{z}}=7.5 \mathrm{~cm}$. Round beam $\beta_{\mathrm{x}}=15 \mathrm{~cm}, \beta_{\mathrm{y}}=15 \mathrm{~cm}$, $\varepsilon_{\mathrm{x}}=\varepsilon_{\mathrm{y}}$. 
FERMILAB-TM-2573-APC

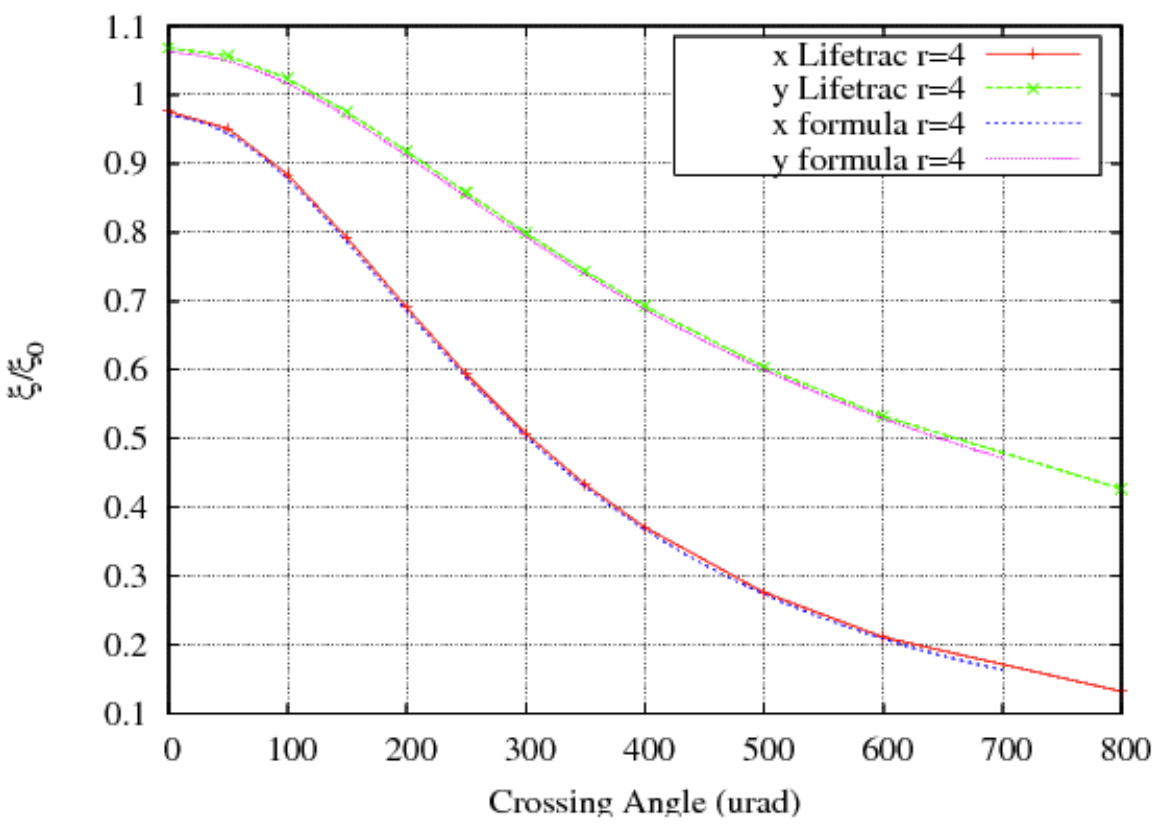

Figure 4: Tune shift factor vs. crossing angle for $\sigma_{\mathrm{z}}=7.5 \mathrm{~cm}$. Flat beam $\beta_{\mathrm{x}}=30 \mathrm{~cm}, \beta_{\mathrm{y}}=7.5 \mathrm{~cm}, \varepsilon_{\mathrm{x}}=\varepsilon_{\mathrm{y}}$.

\section{References}

[1] B. Montague, CERN/ISR-GS/75-36 (1975)

[2] D. Shatilov and M.Zobov, ICFA Beam Dynamics Newsletter No.37, p.99 (2005)

[3] S. Kheifets, DESY PETRA-Note 119, (1976)

[3] K. Hirata, Phys. Rev. Lett. 74, 12 (1993) 